\title{
A CHARACTERISATION OF THE ELLIPSOID
}

\author{
P. W. AITCHISON
}

(Received 24 July 1969)

To Professor Bernhard H. Neumann on his 60th birthday

Communicated by E. S. Barnes

\section{Introduction}

The ellipsoid is characterised among all convex bodies in $n$-dimensional Euclidean space, $R^{n}$, by many different properties. In this paper we give a characterisation which generalizes a number of previous results mentioned in [2], p. 142. The major result will be used, in a paper yet to be published, to prove some results concerning generalizations of the Minkowski theory of reduction of positive definite quadratic forms.

An $n$-dimensional convex body $K$ in $R^{n}$ for $n \geqq 3$ is a closed, bounded, and convex subset of $R^{n}$ which contains exactly $n$ linearly independent points. We call the sets of boundary and interior points of $K$ respectively the frontier and interior of $K$. The notation ' $h K$ ' means the dilation of $K$ about the origin in the ratio $h$. A tac plane of $K$ at a point of the frontier (also known as a support plane) is a hyperplane in $R^{n}$ whose intersection with $K$ includes that point, but no interior points of $K$. These concepts are discussed in detail in [2]. We denote points and vectors in $R^{n}$ as $a, b_{1}$, etcetera, and in particular $O$ is the origin, $\boldsymbol{u}$ always denotes $\boldsymbol{a}$ unit vector, and $\boldsymbol{a} \boldsymbol{b}$ is the chord joining $\boldsymbol{a}$ and $\boldsymbol{b}$.

There are exactly two distinct tac planes of $K$ perpendicular to a vector $\boldsymbol{u}$ and we call the distance between them, the width of $K$ in the direction $\boldsymbol{u}$. A concept of major importance in this paper is that of equivalence of convex bodies. Two $n$ dimensional convex bodies $K_{1}$ and $K_{2}$ are called equivalent if the ratio of the width of $K_{1}$ in the direction $\boldsymbol{u}$ to the width of $K_{2}$ in the direction $\boldsymbol{u}$ is a constant $\boldsymbol{j}$ as $\boldsymbol{u}$ varies in $R^{n}$. This is written in symbols $K_{1} \sim K_{2}$, or more explicitly $K_{1} \sim j K_{2}$.

The major result of this paper is now described (Theorem 1 and Corollary 2). Let $K$ be an $n$-dimensional convex body in $R^{n}$ where $n \geqq 3$. Suppose that any two parallel hyperplanes 'sufficiently close' to a tac plane of $K$ intersect $K$ in equivalent convex bodies. Then $K$ is an ellipsoid. Examples of equivalent convex bodies are two bodies of constant width, or two homothetic convex bedies.

\section{Equivalent convex bodies}

Convex bodies can be discussed very conveniently in terms of their tac func- 
tions. The tac function $H: R^{n} \rightarrow R$ of a convex body $K$ is defined by

$$
\boldsymbol{H}(\boldsymbol{v})=\sup \cdot \boldsymbol{v} \cdot \boldsymbol{x} \text {, where } \boldsymbol{x} \in K .
$$

The properties of this function are discussed in detail in [2] under the name 'Stützfunktion'. We define the outer normal $\boldsymbol{u}$ of a tac plane $U$ of $K$ as the unit vector perpendicular to $U$ so that $\boldsymbol{u}$ is directed away from $K$ when $\boldsymbol{u}$ is translated to a point of contact of $U$ with $K$. It easily follows that if $O$ is in the interior of $K$ then $H(\boldsymbol{u})$ is the distance from $\boldsymbol{O}$ to the tac plane $U$ with outer normal $\boldsymbol{u}$, and so $H(\boldsymbol{u})+H(-\boldsymbol{u})$ is the width of $K$ in the direction $\boldsymbol{u}$. From now on we assume $\boldsymbol{O}$ is in the interior of $K$.

If $K_{1}$ and $K_{2}$ are convex bodies with tac functions $H_{1}$ and $H_{2}$ then we now see that $K_{1} \sim j K_{2}$ if, and only if,

$$
H_{1}(\boldsymbol{u})+H_{1}(-\boldsymbol{u})=j\left(H_{2}(\boldsymbol{u})+H_{2}(-\boldsymbol{u})\right)
$$

for each $u \in R^{n}$. In Lemma 1 we give another 'characterisation' of equivalence. The result of Lemma 1 is probably well-known but no reference is available, though the 'only if' part is in [6], p. 293 for example. It is "easily generalized to $R^{n}$. A tac plane of $K$ is called regular if it has only a single point in common with $K$.

Lemma 1. Let $K_{1}$ and $K_{2}$ be two 2-dimensional convex bodies with tac functions $H_{1}$ and $H_{2}$. Let $U_{1}$ and $V_{1}$ be tac planes of $K_{1}$ at $a_{1}$ and $b_{1}$ both perpendicular to $v$ and $U_{2}$ and $V_{2}$ similarly for $K_{2}$ at $a_{2}$ and $b_{2}$ as shown in Diagram 1. Then for some $j$ we have $K_{1} \sim j K_{2}$ if, and only if, $\boldsymbol{a}_{1} \boldsymbol{b}_{1}$ is parallel to $\boldsymbol{a}_{2} \boldsymbol{b}_{2}$ for any choice of $\boldsymbol{v}$ for which $U_{1}, V_{1}, U_{2}$, and $V_{2}$ are all regular (in these cases $a_{1}, b_{1}, a_{2}$, and $b_{2}$ are uniquely defined).
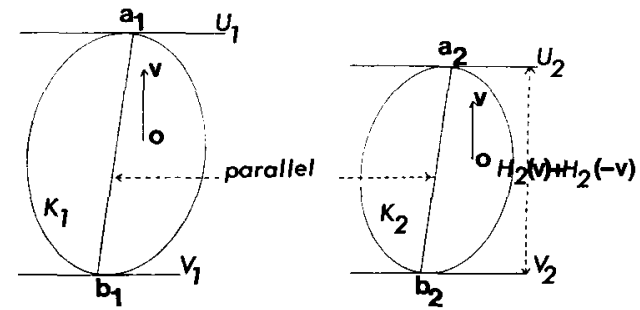

Diagram 1

Proof. The tac planes $U_{1}, V_{1}, U_{2}$, and $V_{2}$ must all be regular for all $v$ in $R^{2}$ except possibly a set of measure zero (on this see [2], section 9). We assume in the following that $v$ is chosen so that the four tac planes are regular. $D_{i}$ denotes the partial derivative with respect to the $i$ 'th variable.

If we denote the components of $a_{1}$ by $a_{1 i}$, for $i=1,2$, and similarly for $a_{2}$, $\boldsymbol{b}_{1}$, and $\boldsymbol{b}_{2}$, then according to [2], p. 24, 26 the partial derivatives of $H_{1}$ and $H_{2}$ exist at $v$, and for $i=1,2$ and $j=1,2$,

$$
D_{i} H_{j}(v)=a_{j i} \text { and } D_{i} H_{j}(-v)=b_{j i} \text {. }
$$


It is clear that the width of $K_{j}$ in the direction $v$, for $j=1,2,\left(H_{j}\right.$ is homogeneous of degree one) is

$$
\left(\boldsymbol{a}_{j}-\boldsymbol{b}_{j}\right) \cdot \boldsymbol{v}=H_{j}(\boldsymbol{v})+H_{j}(-\boldsymbol{v}) .
$$

Now let us assume that $\boldsymbol{a}_{1} \boldsymbol{b}_{1}$ is parallel to $\boldsymbol{a}_{2} \boldsymbol{b}_{2}$ whenever $U_{1}, V_{1}, U_{2}$, and $V_{2}$ are all regular. Then for the regular cases there is a function $J$ so that

$$
\left(a_{1}-b_{1}\right)=J(v)\left(a_{2}-b_{2}\right)
$$

or in components, for $i=1,2$,

By (1) this becomes

$$
\left(a_{1 i}-b_{1 i}\right)=J(v)\left(a_{2 i}-b_{2 i}\right) \text {. }
$$

$$
D_{i}\left(H_{1}(v)-H_{1}(-v)\right)=J(v) D_{i}\left(H_{2}(v)-H_{2}(-v)\right) .
$$

If we take the scalar product of both sides of equation (3) by $v$ and use (2), then we find

$$
H_{1}(\mathfrak{v})+H_{1}(-\mathfrak{v})=J(\mathfrak{v})\left(H_{2}(\mathfrak{v})+H_{2}(-\mathfrak{v})\right) .
$$

This shows that $J$ is differentiable when $\boldsymbol{v} \neq \boldsymbol{O}$ and if we take the $i$ th partial derivative of both sides of equation (5) then we find using (4) that

$$
D_{i} J(v)=0 \text { for } i=1,2 .
$$

This result holds for all $v$ except for a set of measure zero. Consider $J$ to be defined for all $v \neq O$ by (5). A simple analysis argument using (6) and the absolute continuity of $H_{1}$ and $H_{2}$ shows that $J$ is a constant $j$ on any set of points of $R^{n}$ which excludes a neighbourhood of $\boldsymbol{O}$ (the one-dimensional case of this result is well known and is given in [7], p. 90 for example). Hence we deduce from (5) that $K_{1} \sim j K_{2}$ as required.

On the other hand, we now assume that $K_{1} \sim j K_{2}$ for some constant $j>0$, so that

$$
H_{1}(v)+H_{1}(-v)=j\left(H_{2}(v)+H_{2}(-v)\right) .
$$

In the regular cases, the partial derivatives of $H_{1}$ and $H_{2}$ exist according to [2], p. 24, 26. Hence the differentiation of both sides of (7), together with (1), gives, for $i=1,2$,

Therefore

$$
a_{1 i}-b_{1 i}=j\left(a_{2 i}-b_{2 i}\right)
$$

$$
a_{1}-b_{1}=j\left(a_{2}-b_{2}\right),
$$

so that $a_{1} b_{1}$ is parallel to $a_{2} b_{2}$. This completes the proof.

One further simple result on equivalence is needed.

Lemma 2. Let $K_{1}$ and $K_{2}$ be two 2-dimensional convex bodies with $K_{1} \sim j K_{2}$. For $u \in R^{2}$, let the total lengths of all straight segments parallel to $u$ on the bounda- 
ries of $K_{1}$ and $K_{2}$ be $h_{1}$ and $h_{2}$ respectively. Then $h_{1}=j h_{2}$. In particular, if both tac planes of $K_{1}$ parallel to $\boldsymbol{u}$ are regular then so are the corresponding tac planes of $K_{2}$.

The result is well-known and follows from Lemma 1 and a result in [2], p. 31.

\section{A new characterisation of the ellipsoid}

We first state an important characterisation of the ellipsoid due to Blaschke, in Lemma 3. A proof is given of the $n$-dimensional generalization in [3], p. 93.

LemMA 3. Let $K$ be a 3-dimensional convex body. Let $T(\boldsymbol{u})$ be the union set of all lines parallel to $\boldsymbol{u}$ which intersect $K$ but not its interior. If the set

$$
C(\boldsymbol{u})=T(\boldsymbol{u}) \cap K
$$

lies in a plane for each $u \in R^{3}$ then $K$ is an ellipsoid.

We can now state the major result of this paper. Further results on ellipsoids are given in Corollaries 2 and 3 in the next section.

THEOREM 1. Let $K$ be a 3-dimensional convex body in $R^{3}$ with $\boldsymbol{O}$ in its interior and with all its tac planes regular. Let $h$ be a constant with $0<h<1$. Suppose that the 2-dimensional convex bodies $W_{1} \cap K$ and $W_{2} \cap K$ are equivalent for all pairs of parallel planes $W_{1}$ and $W_{2}$ both on the same side of $\boldsymbol{O}$, provided $W_{1}$ and $W_{2}$ intersect the interior of $K$ but not the interior of $h K$. Then $K$ is an ellipsoid.

The condition that $O$ is in the interior of $K$ is not really necessary, but it is convenient for the statement and proof of the theorem. The condition ' $W_{1}$ and $W_{2}$ do not intersect the interior of $h K$ ' is only used to show that we need only consider such planes which are 'close to the boundary' of $K$. We might have said instead that 'there is an $\varepsilon>0$ so that $W_{1}$ and $W_{2}$ are within a perpendicular distance $\varepsilon$ of a tac plane of $K$ which is parallel to $W_{1}$ and $W_{2}$.

The following simple Corollary can be seen immediately and it incorporates some previous results in [2], p. 142.

COROllary 1. Let $K$ be a 3-dimensional convex body. If $W_{1} \cap K$ and $W_{2} \cap K$ are equivalent whenever $W_{1}$ and $W_{2}$ are two parallel planes intersecting the interior of $K$, then $K$ is an ellipsoid.

The proof of Theorem 1 is long and it is given in the series of Lemmas 4-10 to each of which the hypothesis of the Theorem applies. The aim of the proof is to show that the set $C(\boldsymbol{u})$, defined in Lemmas 3 , lies in a plane, so that Lemma 3 may be applied to show $K$ is an ellipsoid.

Lemma 4. Let $C(\boldsymbol{u})$ and $T(\boldsymbol{u})$ be defined as in Lemma 3.

(a) Any plane $W$ parallel to the vector $\boldsymbol{u}$ which intersects the interior of $K$ will intersect $C(\boldsymbol{u})$ in two distinct points.

(b) $C(\boldsymbol{u})$ is a simple closed curve in $R^{3}$. 
Proof. (a). $T(\boldsymbol{u})$ is a convex cylinder, and $W$ clearly intersects $T(\boldsymbol{u})$ in two distinct generators of $T(\boldsymbol{u})$. These two generators must lie in distinct tac planes of $K$, and because of the regularity of the tac planes they each have a single point in common with $K$ and so with $C(\boldsymbol{u})$.

(b) If $Q$ is the line through $O$ parallel to the vector $\boldsymbol{u}$ then from the proof of (a) it follows that any half-plane bounded by $Q$ has a single point in common with $C(u)$. Choose any one such half-plane and designate it $V(O)$ and let $V(s)$ designate the half-plane making an angle $s$ with $V(O)$, for $0 \leqq s \leqq 2 \pi$. Let $a(s)$ be the unique point of $C(\boldsymbol{u})$ in $V(s)$. Now

$$
C(\boldsymbol{u})=\{a(s) \mid 0 \leqq s<2 \pi\},
$$

while $\boldsymbol{a}(O)=\boldsymbol{a}(2 \pi)$ and $\boldsymbol{a}(s) \neq \boldsymbol{a}(t)$ if $0 \leqq s<t<2 \pi$. We must show $\boldsymbol{a}(s)$ is a continuous function of $s . C(\boldsymbol{u})$ is a closed set since it is the intersection of the closed sets $K$ and $T(\boldsymbol{u})$. If $\left\{s_{i}\right\}$ is a sequence with $s_{\boldsymbol{i}} \rightarrow s$ then $\left\{\boldsymbol{a}\left(s_{i}\right)\right\}$ must have at least one limit point which can only be in $V(s)$. Since $C(\boldsymbol{u})$ is closed this limit point must be $\boldsymbol{a}(s)$. This completes the proof.

LemMa 5. Let $W_{1}$ and $W_{2}$ be any two planes as in the hypothesis of Theorem 1 so that $W_{1} \cap K$ is equivalent to $W_{2} \cap K$. If $W_{1}$ intersects $C(u)$ in $a_{1}$ and $b_{1}$, and $W_{2}$ intersects $C(\boldsymbol{u})$ in $\boldsymbol{a}_{2}$ and $\boldsymbol{b}_{2}$, then $\boldsymbol{a}_{1} \boldsymbol{b}_{1}$ is parallel to $\boldsymbol{a}_{2} \boldsymbol{b}_{2}$.

Proof. There is a line $U_{1}$ in $W_{1}$ parallel to $\boldsymbol{u}$ which intersects $K$ in $a_{1}$, and similarly lines $V_{1}$ in $W_{1}$ and $U_{2}$ and $V_{2}$ in $W_{2}$ intersecting $K$ in $\boldsymbol{b}_{1}, \boldsymbol{a}_{2}$, and $\boldsymbol{b}_{2}$. Clearly $U_{1}$ and $V_{1}$ are parallel tac planes of the 2-dimensional convex body $W_{1} \cap K$ at $a_{1}$ and $b_{1}$, and $U_{2}$ and $V_{2}$ similarly for $W_{2} \cap K$ at $a_{2}$ and $b_{2}$. Hence by Lemma 1 the result follows immediately.

In order to simplify the following proofs we now construct a function $f:[0,2 \pi] \rightarrow R$. By an orthogonal transformation of $R^{n}$ we can make $\boldsymbol{u}=(0,0,1)$. Then each point of $C(\boldsymbol{u})$ is the point of contact with $K$ of a tac plane with an outer normal $(\cos r, \sin r, 0)$ for some $r \in[0,2 \pi]$, and we write this point $f(r)$. We easily see the following: $\boldsymbol{f}$ is continuous, $\boldsymbol{f}$ is a surjection, $\boldsymbol{f}$ is injective except at most countably many points of $C(\boldsymbol{u})$ where $K$ has more than one tac plane; $f$ induces the ordering of $[0,2 \pi]$ on $C(\boldsymbol{u})$ (excluding $f(0)$ ). We loose no generality in assuming $f$ is injective at $r=0$. Notice that the tac plane of $K$ at $f(r)$ makes an angle $r$ with the tac plane of $K$ at $f(O)$.

Notice also that a plane perpendicular to $(\cos r, \sin r, 0)$ cuts $C(\boldsymbol{u})$ not at all, or in one of $f(r), f(r \pm \pi)$, or in two points $f(s)$ and $f\left(s^{\prime}\right)$. In the last case we call $\boldsymbol{f}(s) f\left(s^{\prime}\right)$ an $r$-chord. Clearly there is a unique $r$-chord through each point of $C(\boldsymbol{u})$ other than $f(r)$ or $f(r \pm \pi)$.

We now prove some continuity properties of $r$-chords.

LEMMA 6. (a) We may define $r$ as a continuous function of $s$ and $s^{\prime}\left(s<s^{\prime}\right)$ by $f(s) f\left(s^{\prime}\right)$ being an $r$-chord with $s<r<s^{\prime}$, in the cases when this $r$-chord is defined. If $s^{\prime}$ is fixed then $r$ is a monotonic increasing function of $s$. 
(b) If $f\left(s^{\prime}\right)$ is defined as a function of $r$ and $s$ by $f(s) f\left(s^{\prime}\right)$ being an $r$-chord then the function is continuous.

Proof. (a) Let $f(s) f\left(s^{\prime}\right)$ be a $q$-chord with say $q<\pi$, then the plane $W$ parallel to $\boldsymbol{u}$ and containing $f(s) f\left(s^{\prime}\right)$ separates the tac planes $T_{1}$ and $T_{2}$ of $K$ at $\boldsymbol{f}(q)$ and $\boldsymbol{f}(q+\pi)$. In addition $W$ separates $C(\boldsymbol{u})$ into two parts, one of which is $\left\{f(x) \mid s<x<s^{\prime}\right\}$ and so we define $r$ to be whichever of $q$ or $q+\pi$ lies in this part. Since $f$ is continuous, the plane $W$ containing $f(s)$ and $f\left(s^{\prime}\right)$ must vary continuously with $s$ and $s^{\prime}$, and therefore also the planes $T$ and $T^{\prime}$. Yet $T$ and $T^{\prime}$ make angles $q$ and $q+\pi$ with a fixed plane parallel to $\boldsymbol{u}$, so it follows that $r$ also varies continuously with $s$ and $s^{\prime}$.

If $s^{\prime}$ is fixed and $s$ decreases monotonically then $W$ and so $T_{1}$ and $T_{2}$ must rotate monotonically, and they must rotate in the sense of decreasing $r$ (for otherwise $r$ would not stay in the interval $\left(s, s^{\prime}\right)$. This completes part (a).

(b) The plane $W$ varies continuously with $r$ and $s$, and therefore so does its second point of intersection with $C(u)$, namely $f\left(s^{\prime}\right)$. This completes the proof.

We next describe an important property-of $r$-chords.

Lemma 7. For each $r \in(0,2 \pi)$ there is a neighborhood $\left(p, p^{\prime}\right) \subset(0,2 \pi)$, designated $N(r)$, satisfying:

(a) $f(r)$ is in the interior of $\left\{f(x) \mid p<x<p^{\prime}\right\}$ on $C(u)$;

(b) $\boldsymbol{f}(p)$ and $\boldsymbol{f}\left(p^{\prime}\right)$ are continuous functions of $r$;

(c) if $s, t \in N(r)$ and $f(s) f\left(s^{\prime}\right)$ and $f(t) f\left(t^{\prime}\right)$ are r-chords then they are parallel.

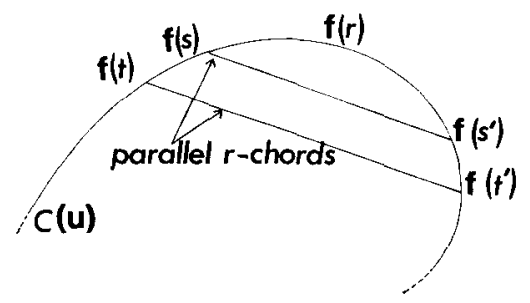

Diagram 2

Proof. Let $W$ be a plane perpendicular to $(\cos r, \sin r, 0)$ intersecting $C(\boldsymbol{u})$ in $\boldsymbol{f}(q)$ and $\boldsymbol{f}\left(q^{\prime}\right)$ with $q<q^{\prime}$, (so $\boldsymbol{f}(q) \boldsymbol{f}\left(q^{\prime}\right)$ is an $\boldsymbol{r}$-chord). Then $W$ divides $C(\boldsymbol{u})$ into two parts one of which is $\left\{f(x) \mid q<x<q^{\prime}\right\}$, and then the other part contains $f(0)$. However $f(r) \neq f(0)$ since $r \neq 0$ and $f$ is injective at $r=0$, thus we can choose $W$ so that it separates $f(0)$ and $f(r)$ and then it follows that $q<r<q^{\prime}$. We choose $p$ and $p^{\prime}$ so that $f(p) f\left(p^{\prime}\right)$ is an $r$-chord and $p$ is the minimum value of $q$ with this property $q<r<q^{\prime}$, subject also to the condition that $W$ does not intersect the interior of $h K$ ( $h$ as in the hypothesis of Theorem 1). This defines $p$ and $p^{\prime}$ uniquely and $f(p) \neq f(r)$ (since $h<1$ ) so that (a) is satisfied. Property (c) follows from Lemma 5 and the hypothesis of the theorem with $N(r)=\left(p, p^{\prime}\right)$. We still have to establish property (b). 
Let $W$ now be the plane perpendicular to $(\cos r, \sin r, 0)$ containing $f(p)$ and $f\left(p^{\prime}\right)$. It is clear from the construction of $f(p)$ and $f\left(p^{\prime}\right)$ that either $W$ contains $f(0)$ (in which case either $p=0$ or $p^{\prime}=2 \pi$ ) or if not, then $W$ is a tac plane of $h K$. In the first case the continuity follows from Lemma $6(\mathrm{~b})$ (since one end point of $f(p) f\left(p^{\prime}\right)$ is fixed). In the second case the tac plane $W$ varies continuously with $r$ and therefore the points of intersection of $W$ with $C(\boldsymbol{u})$, namely $\boldsymbol{f}(p)$ and $f\left(p^{\prime}\right)$, vary continuously with $r$. This completes the proof.

We next show that $C(u)$ is a plane curve and thus complete the proof of Theorem 1 by applying Lemma 3 .

LeMma 8. $C(\boldsymbol{u})$ is a plane curve.

Proof. Let us take a closed subinterval of $(0,2 \pi)$, say $[a, b]$. Define the function $D:[a, b] \rightarrow R$ by

$$
D: r \mapsto \min .\left(|f(r)-f(p)|,\left|f(r)-f\left(p^{\prime}\right)\right|\right),
$$

where $N(r)=\left(p, p^{\prime}\right)$. Then by Lemma 7 (a) and (b), $D$ is strictly positive and continuous, so there exists a constant $m>0$ so that $D(r)>m$ tor $r \in[a, b]$.

Now we choose an open subinterval of $[a, b], I$ say, satisfying $|f(x)-f(y)|$ $\leqq m$ whenever $x, y \in I$, and also so that the set $f(I)$ is more then one point. We will show that the set $f(I)$ lies in a plane. Notice the following property of $I$ which follows from the definition (1) and definitions of $m$ and $I$,

$$
N(r) \supset I \text { for } r \in I \text {. }
$$

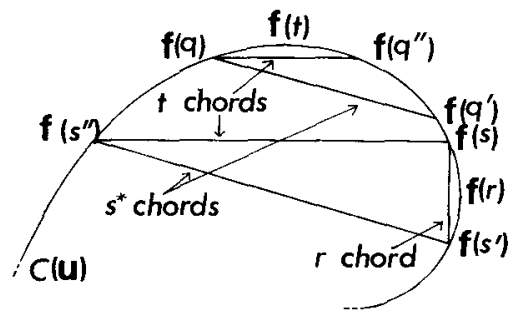

Diagram 3

Let $r$ and $t \in I$ and let $f(s) f\left(s^{\prime}\right)$ be an $r$-chord with $s \in I$ and $f(s) f\left(s^{\prime \prime}\right)$ a $t$-chord as in Diagram 3. As $s \rightarrow r$ we have $s^{\prime} \rightarrow r$ by Lemma 6(b), and therefore, since $I$ is open, there is an $s_{0} \in I$ so that $s^{\prime} \in I$ whenever $s_{0}<s<s_{0}^{\prime}$. Let $s^{*}$ be so that $f\left(s^{\prime}\right) f\left(s^{\prime \prime}\right)$ is an $s^{*}$-chord. By Lemmas 6(a) and (b), $s^{*}$ is a continuous function of $s$ and so $s^{*} \rightarrow t$ as $s \rightarrow r$. Hence there is an $s_{1}$ with $s_{1} \geqq s_{0}$ so that $s^{*} \in I$ whenever $s_{1}<s<s_{1}^{\prime}$ (and we can also easily ensure $f\left(s_{1}\right) \neq f(r)$ ).

All of the $r$-chords $f(s) f\left(s^{\prime}\right)$ are parallel for $s_{1}<s<s_{1}^{\prime}$ by Lemma 7 since $s \in I$ and $N(r) \supset I$ by (2). Similarly the $t$-chords $f(s) f\left(s^{\prime \prime}\right)$ are parallel for $s_{1}<s<s_{1}^{\prime}$. Hence the $s^{*}$-chords $f\left(s^{\prime}\right) f\left(s^{\prime \prime}\right)$, for $s_{1}<s<s_{1}^{\prime}$, are parallel to the 
plane $U$ through $\boldsymbol{O}$ spanned by $f(s)-f\left(s^{\prime}\right)$ and $f(s)-f\left(s^{\prime \prime}\right)$ for any such $s$.

Let $q \in I$ and $f(q) f\left(q^{\prime}\right)$ be the $s^{*}$-chord through $f(q)$. Then $f(q) f\left(q^{\prime}\right)$ is parallel to $f\left(s^{\prime}\right) f\left(s^{\prime \prime}\right)$, for $s_{1}<s<s_{1}^{\prime}$, by Lemma 7 , since in these cases $s^{\prime} \in I$ and $N\left(s^{*}\right) \supset I$. Hence all of these points $f\left(q^{\prime}\right)$ lie in the plane $V$ through $f(q)$ parallel to $U$. By Lemma $6(\mathrm{~b})$ these points $f\left(q^{\prime}\right)$ comprise a proper interval on $\boldsymbol{C}(\boldsymbol{u})$ containing $\boldsymbol{f}\left(q^{\prime \prime}\right)$ in its interior, where $\boldsymbol{f}(q) \boldsymbol{f}\left(q^{\prime \prime}\right)$ is a $t$-chord. Hence by an appropriate choice of $q$ and $t$ we can show that any point of $I$, is in the interior of an interval of points of $C(\boldsymbol{u})$ lying in a plane. Therefore all of the points $f(x)$, for $x \in I$, lie in a plane.

It easily follows from the arbitrary choice of the subinterval $I$ of $[a, b]$ and of the interval $[a, b]$ itselt that all of the points $f(x)$, for $x \in(0,2 \pi)$, lie in a plane and so $C(\boldsymbol{u})$ is a plane curve. This completes Lemma 8 .

Theorem 1 now follows trom Lemmas 3 and 8.

\section{Further results on ellipsoids}

The result of section 3 , namely Theorem 1 , is now generalized in several ways. In Corollary 2 we extend the result to $R^{n}$ without difficulty.

Corollary 2. Let $K$ be an $n$-dimensional convex body in $R^{n}$ for $n \geqq 3$ with $O$ in its interior and with all of its tac planes regular. Let $u$ be a constant with $0<h<1$. Suppose that the $(n-1)$-dimensional convex bodies $\left(W_{1} \cap K\right)$ and $\left(W_{2} \cap K\right)$ are equivalent for all parallel hyperplanes $W_{1}$ and $W_{2}$ both on the same side of $O$, provided $W_{1}$ and $W_{2}$ intersect the interior of $K$ but not the interior of $h K$. Then $K$ is an ellipsoid.

Proof. In the case $n=3$ this is Theorem 1. The proof uses induction on $n$. Let $K$ be an $n$-dimensional convex body satisfying the hypothesis and assume the result is true for $(n-1)$-dimensional convex bodies. It is then easy to show that if $U$ is any hyperplane through $\boldsymbol{O}$ then the $(n-1)$-dimensional convex body $(U \cap K)$ is an ellipsoid. It follows that $K$ is an ellipsoid (see [3], p. 91). This completes the proof.

The condition that the tac planes be regular is not necessary and we now show that the hypothesis of Corollary 2 can be restated in terms of the regular tac planes of an arbitrary convex body $K$. The hypothesis of the next result is stated in a slightly different form from Corollary 2 for convenience in the proof.

Corollary 3. Let $K$ be an $n$-dimensional convex body in $R^{n}$ with $\boldsymbol{O}$ in its interior and $n \geqq 3$. Let $h$ be a constant with $0<h<1$. Suppose that the $(n-1)$-dimensional convex bodies $\left(W_{1} \cap K\right)$ and $\left(W_{2} \cap K\right)$ are equivalent whenever there are $\boldsymbol{u}, h_{1}$, and $h_{2}$ with $h \leqq h_{1}<1$, and $h \leqq h_{2}<1$, and $W_{1}$ and $W_{2}$ are regular tac planes of $h_{1} K$ and $h_{2} K$, respectively, both with outer normal $u$. Then $K$ is an ellipsoid.

Proof. We prove that every tac plane of $K$ is regular, in which case the result 
follows from Corollary 1 . We prove this regularity only when $n=3$ since the general case follows by a simple induction argument.

As a first step we show that the equivalence condition of the hypothesis can be extended to include planes $W_{1}$ and $W_{2}$ which are not regular tac planes of $h_{1} K$ and $h_{2} K$. We can certainly find a sequence $\left\{\boldsymbol{u}_{i}\right\}$ with $\boldsymbol{u}_{\boldsymbol{i}} \rightarrow \boldsymbol{u}$ as $i \rightarrow \infty$ so that the tac planes $W_{1 i}$ of $h_{1} K$ and $W_{2 i}$ of $h_{2} K$ with outer normals $\boldsymbol{u}_{i}$, for each $i$, are regular. This is because the normals of non-regular tac planes form a set of measure zero in $R^{3}$ (see [2], section 9). For each $i$ the hypothesis shows that $\left(W_{1 i} \cap K\right)$ is equivalent to $\left(W_{2 i} \cap K\right)$. However $\left(W_{1} \cap K\right)$ is the limit as $i \rightarrow \infty$ of the convex bodies $\left(W_{1 i} \cap K\right)$ in the sense that the boundary points of $\left(W_{1 i} \cap K\right)$ become uniformly arbitrarily close to those of $\left(W_{1} \cap K\right)$ as $i \rightarrow \infty$ and vice-versa (an accurate description of this can be given in terms of a metric on convex bodies as given in [5] p. 235 for example). Using the definition of equivalence, it follows that $\left(W_{1} \cap K\right)$ is equivalent to $\left(W_{2} \cap K\right)$.

We now show that no tac plane of $K$ can intersect $K$ in just a segment. Suppose a tac plane with outer normal $\boldsymbol{u}$ intersects $K$ in just a segment. Let $W_{1}$ be the tac plane of $h_{1} K$ with outer normal $u$. If we let $h_{1} \rightarrow 1$ (from below) then we can easily show that the ratio of the minimum to maximum width of $\left(W_{1} \cap K\right)$ must approach zero since the set $\left(W_{1} \cap K\right)$ must have the segment as its limit. This is incompatible with the equivalence condition of the hypothesis. Hence any tac plane of $K$ intersects $K$ in a single point or a proper plane face.

Suppose that a tac plane $T$ intersects $K$ in a plane face, and $T^{\prime}$ is the other tac plane of $K$ parallel to $T$. Let $D=T \cap K, D^{\prime}=T^{\prime} \cap K$ (considered as 2-dimensional convex bodies) where $D^{\prime}$ may be a single point, and choose any proper chord $a_{1} a_{2}$ of $D$. We divide the argument into two cases: (i) every tac plane of $K$ parallel to $a_{1} a_{2}$ intersects $D$ or $D^{\prime}$; (ii) there is a tac plane $W$ of $K$ parallel to $a_{1} a_{2}$ which does not intersect $D$ or $D^{\prime}$.

In case (i) we can clearly find a tac plane $W$ of $K$ parallel to $a_{1} a_{2}$ which intersects both $D$ and $D^{\prime}$. Then $W \cap K$ must contain a segment which does not lie in $T$ or $T^{\prime}$. It follows from the foregoing that this segment lies in a plane face of $K$ parallel to $a_{1} a_{2}$ and distinct from $D$ and $D^{\prime}$.

In case (ii) let $W_{1}$ be the tac plane of $h K$ with the same outer normal as $W$. We can show by a simple continuity argument that $W$ may be rechosen parallel to $a_{1} a_{2}$ and still distinct from $D$ and $D^{\prime}$, so that $W_{1}$ intersects the interior of $D$. In this case we choose $W_{2}$ to be another plane parallel to $W$ lying strictly between $W$ and $W_{1}$ which does not intersect $D$ or $D^{\prime}$. Clearly $W_{1}$ and $W_{2}$ satisfy the equivalence condition of the hypothesis so that $\left(W_{1} \cap K\right)$ is equivalent to $\left(W_{2} \cap K\right)$. However the frontier of $\left(W_{1} \cap K\right)$ contains a proper segment parallel to $a_{1} a_{2}$ (in its intersection with $D)$, and so by Lemma 2 the frontier of $\left(W_{2} \cap K\right)$ also contains a proper segment parallel to $a_{1} a_{2}$. This segment of $\left(W_{2} \cap K\right)$ does not lie in $D$ or $D^{\prime}$ and so, as we showed previously, it lies in a plane face of $K$ distinct from $D$ and $D^{\prime}$. 
In both cases (i) and (ii) we deduce that there is a plane face $F_{1}$ of $K$ parallel to $a_{1} a_{2}$ and distinct from $D$ and $D^{\prime}$. For another chord $b_{1} b_{2}$ of $D$ which is not parallel to $a_{1} a_{2}$ we find another plane face $F_{2}$ parallel to $b_{1} b_{2}$, which is distinct from $D$ and $D^{\prime}$. However $F_{1}$ and $F_{2}$ are distinct since the only plane taces of $K$ parallel to $a_{1} a_{2}$ and $b_{1} b_{2}$ are $D$ and $D^{\prime}$. It tollows that there are uncountably many different plane faces of $K$ since there are uncountably many mutually nonparallel chords of $D$. However $K$ cannot have uncountably many plane faces, and so this is a contradiction of the supposition that it had one plane face. Hence all tac planes of $K$ are regular, and this completes the proof of Corollary 3.

Another possible generalization of Theorem 1 would be to relax the condition that $h$ be a constant and define $h$ locally for each set of parallel planes. More exactly we would consider the following condition: $K$ is an n-dimensional convex body in $R^{n}$, for $n \geqq 3$, with $\boldsymbol{O}$ in its interior. For each $\boldsymbol{u}$ there is a $h(\boldsymbol{u})$ with $0<h(\boldsymbol{u})<1$. $T(\boldsymbol{u})$ is the tac plane of $K$ with outer normal $\boldsymbol{u}$. Then for each $\boldsymbol{u}, W_{1} \cap K$ is equivalent to $W_{2} \cap K$ whenever $W_{1}$ and $W_{2}$ are any two hyperplanes, both parallel to, and on the same side of $\boldsymbol{O}$, as $T(\boldsymbol{u})$, where $W_{1}$ and $W_{2}$,intersect the interior of $K$ but not the interior of $h(u) K$.

Rather surprisingly $K$ need not be an ellipsoid now since the body $K$ composed of a hemisphere and half-ellipsoid given by

$$
\begin{aligned}
\frac{1}{4} x_{1}^{2}+x_{2}^{2}+x_{3}^{2} & \leqq 1 \text { for } x_{1} \geqq 0 \\
x_{1}^{2}+x_{2}^{2}+x_{3}^{2} & \leqq 1 \text { for } 0 \geqq x_{1}
\end{aligned}
$$

satisfies this condition. There is strong evidence that any $K$ satisfying this condition must be composed of 'pieces' of second order surfaces.

\section{References}

[1] T. M. Apostol, Mathematical Analysis, A Modern Approach to Advanced Calculus (AddisonWesley, Reading, Massachusetts, 1957).

[2] T. Bonnesen, and W. Fenchel, Theorie der konvexen Körper (Reprint by Chelsea, New York, 1948).

[3] H. Busemann, Geometry of Geodesics (Academic Press, New York, 1955).

[4] R. E. Edwards, Functional Analysis: Theory and Applications (Holt, Rinehart and Winston, New York, 1965).

[5] B. Grünbaum, Measures of Symmetry for Convex Sets (Proc. Sympos. Pure Math. VII, Convexity, 233-270. Amer. Math. Soc., 1963).

[6] P. C. Hammer, Convex curves of constant Minkowski breadth (Proc. Sympos. Pure Math VII, Convexity, 291-304. Amer. Math. Soc., 1963).

[7] H. L. Royden, Real Analysis (Macmillan, New York, 1963).

[8] W. Süss, 'Kennzeichende Eigenschaften der Kugel als Folgerung eines Brouwerschen Fixpunktsatzes', Comm. Math. Helv. 20 (1947), 61-64.

Australian National University

Canberra 